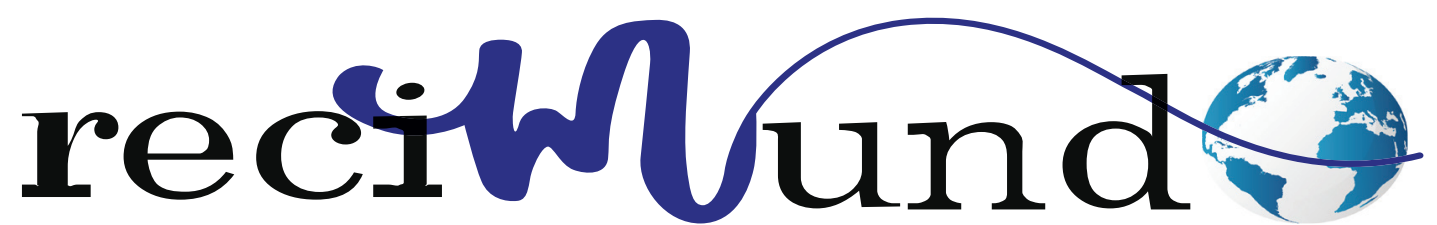

Revista Científica Mundo de la Investigación y el Conocimiento

DOI: $10.26820 /$ recimundo/5.(1).enero.2021.42-49

URL: http://recimundo.com/index.php/es/article/view/981

EDITORIAL: Saberes del Conocimiento

REVISTA: RECIMUNDO

ISSN: 2588-073X

TIPO DE INVESTIGACIÓN: Artículo de Revisión

CÓDIGO UNESCO: 3205 Medicina Interna

PAGINAS: 42-49

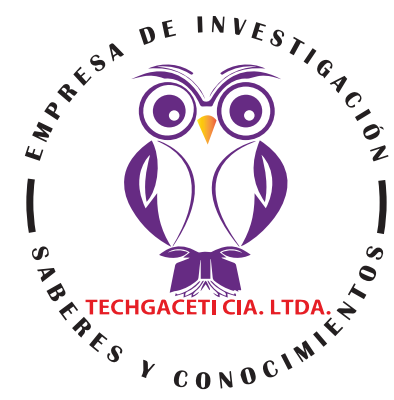

\title{
Aspectos fisiopatológicos en la hipertensión portal
}

Pathophysiological aspects in portal hypertension

Aspectos fisiopatológicos da hipertensão portal

Luis Adrián Muñoz Andrade'; Ana Julia Mera Sabando2; Eva Yulissa López Álvarez;;

Johan Alexander Tóala Cedeño ${ }^{4}$

\section{RECIBIDO: 28/11/2020 ACEPTADO: 06/12/2020 PUBLICADO: 31/01/2021}

1. Médico; Médico General Institucional del Consejo Nacional Electoral; Quito, Ecuador; munozandradeluis@gmail.com; https://orcid.org/0000-0002-9682-5896

2. Médico Cirujano; Cargo Ocupación Actual: Médico Rural Centro de Salud Frutillo de Rocafuerte; Ecuador; anajulims@ hotmail.com; (iD) https://orcid.org/0000-0001-8608-4716

3. Médico Cirujano; Cargo Ocupación Actual: Médico Rural Centro de Salud 24 de Mayo; Ecuador; evitalo1996@gmail.com; (iD) https://orcid.org/0000-0002-6639-2153

4. Médico General; Cargo Ocupacional: Médico Rural; Ecuador; johan_0310@hotmail.com; (DD https://orcid.org/0000-00033084-8793

CORRESPONDENCIA

Luis Adrián Muñoz Andrade

munozandradeluis@gmail.com

Quito, Ecuador

() RECIMUNDO; Editorial Saberes del Conocimiento, 2021 


\section{RESUMEN}

La hipertensión portal es una enfermedad que generalmente, se presenta en paciente con enfermedades hepáticas crónicas, como la cirrosis. Sin embargo, esta se puede presentar por otros motivos como la hipertensión portal no cirrótica, producida por la esquistosomiasis. Y que se puede clasificar como hipertensión portal prehepática, intrahepática o posthepática. La metodología de la investigación, es una revisión bibliográfica, que se apoyó en medios electrónicos como fuente primaria para la obtención de la información, gracias a medios electrónicos como revistas, páginas web, entre otros. Entre las conclusiones que se pueden derivar de la investigación están, que el estudio de la fisiopatología de la hipertensión portal, ha evolucionado los últimos años. La obstrucción del flujo venoso portal, por los cambios en la arquitectura del hígado y vasodilatación esplanica y sistémica. Hacia un estado hemodinámico, regulado por componentes neurales, celulares y humorales, que actúan de manera endocrina, paracrina y autocrina. En la actualidad hay diferentes tipos de mecanismos de diagnóstico de la hipertensión portal. Uno de los más utilizados y confiables es el gradiente de presión venosa hepática (GPHV), que aunque es un método invasivo, sigue siendo el más sensible y específico, para su diagnóstico. Por otro lado, la elastografía por resonancia magnética, el fibrotest y ARFI, son métodos no invasivos, pero requieren un valor (> $12 \mathrm{~mm} \mathrm{Hg}$ ), para su diagnóstico, lo que la limitan en su uso cotidiano en la evaluación de pacientes con hepatopatías crónicas.

Palabras clave: Fisiopatología, Portal, Hepática, Cirrosis, Resonancia.

\section{ABSTRACT}

Portal hypertension is a disease that generally occurs in patients with chronic liver diseases, such as cirrhosis. However, this can occur for other reasons such as non-cirrhotic portal hypertension, produced by schistosomiasis. And that can be classified as prehepatic, intrahepatic or posthepatic portal hypertension. The research methodology is a bibliographic review, which relied on electronic media as the primary source for obtaining information, thanks to electronic media such as magazines, web pages, among others. Among the conclusions that can be derived from the research are that the study of the pathophysiology of portal hypertension has evolved in recent years. Obstruction of portal venous flow, due to changes in the architecture of the liver and splanic and systemic vasodilation. Towards a hemodynamic state, regulated by neural, cellular, and humoral components, which act endocrine, paracrine, and autocrine. Currently there are different types of diagnostic mechanisms for portal hypertension. One of the most used and reliable is the hepatic venous pressure gradient (GPHV), which, although it is an invasive method, is still the most sensitive and specific for its diagnosis. On the other hand, magnetic resonance elastography, fibrotest and ARFI are non-invasive methods, but they require a value $(>12 \mathrm{~mm} \mathrm{Hg})$ for their diagnosis, which limits their daily use in the evaluation of patients with liver disease. Chronicles.

Keywords: Pathophysiology, Portal, Hepatic, Cirrhosis, Resonance.

\section{RESUMO}

A hipertensão portal é uma doença que geralmente ocorre em pacientes com doenças hepáticas crônicas, tais como cirrose. Entretanto, isto pode ocorrer por outros motivos, como a hipertensão portal não cirrótica, produzida pela esquistossomose. E isso pode ser classificado como hipertensão portal pré-hepática, intra-hepática ou pós-hepática. A metodologia de pesquisa é uma revisão bibliográfica, que contou com meios eletrônicos como fonte primária para a obtenção de informações, graças a meios eletrônicos como revistas, páginas web, entre outros. Entre as conclusões que podem ser derivadas da pesquisa estão que o estudo da fisiopatologia da hipertensão portal evoluiu nos últimos anos. Obstrução do fluxo venoso portal, devido a mudanças na arquitetura do fígado e vasodilatação esplânica e sistêmica. Em direção a um estado hemodinâmico, regulado por componentes neurais, celulares e humorais, que atuam de forma endócrina, parácrina e autócrina. Atualmente existem diferentes tipos de mecanismos de diagnóstico para hipertensão portal. Um dos mais utilizados e confiáveis é o gradiente de pressão venosa hepática (GPHV), que, embora seja um método invasivo, ainda é o mais sensível e específico para seu diagnóstico. Por outro lado, a elastografia por ressonância magnética, fibroteste e ARFI são métodos não invasivos, mas requerem um valor (> $12 \mathrm{~mm} \mathrm{Hg}$ ) para seu diagnóstico, o que limita seu uso diário na avaliação de pacientes com doença hepática. Crónicas.

Palavras-chave: Patofisiologia, Portal, Hepática, Cirrose, Ressonância. 


\section{Introducción}

La hipertensión portal es un síndrome muy frecuente caracterizado por un aumento patológico de la presión hidrostática en el territorio venoso portal. La presión portal normal es entre 5-10 mm de Hg. La hipertensión portal es definida cuando la presión de enclavamiento a nivel de venas hepáticas o la presión en vena porta por punción directa es superior en 5 $\mathrm{mm}$ de Hg a la presión en vena cava inferior (gradiente porto sistémico superior a 5mm de Hg) o la presión venosa esplénica superior a 15mm de Hg (Álvarez, 2002, pág. 46).

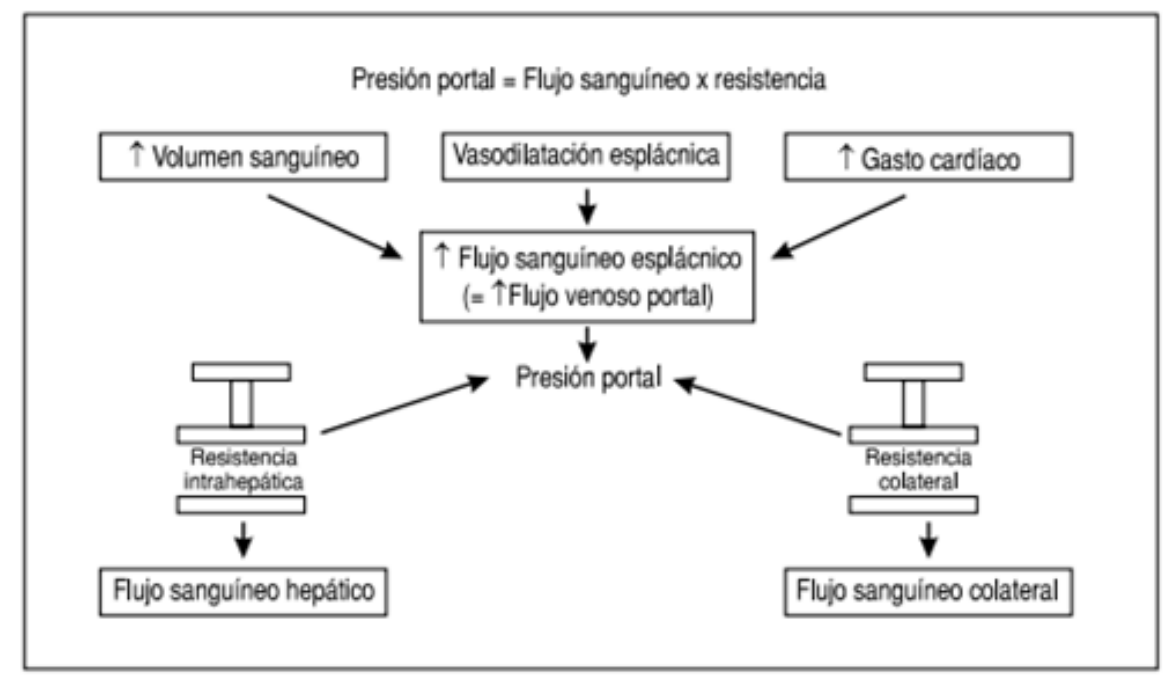

Figura 1. Principios hemodinámicos de la presión portal

Fuente: (Montaño-Loza \& Meza-Junco, 2005)

Una elevación encima de los $10 \mathrm{mmHg}$ ya es considerada como HP y se expresa clínicamente a partir de los $12 \mathrm{mmHg}$. Otra definición de HP es el gradiente de presión más de $6 \mathrm{mmHg}$ entre la VP y la vena cava inferior o una presión venosa esplénica mayor a 15 mmHg.2 (Ibarrola-Calleja, Núñez, Rodríguez, \& Ordóñez-Céspedes, 2011, pág. 83).

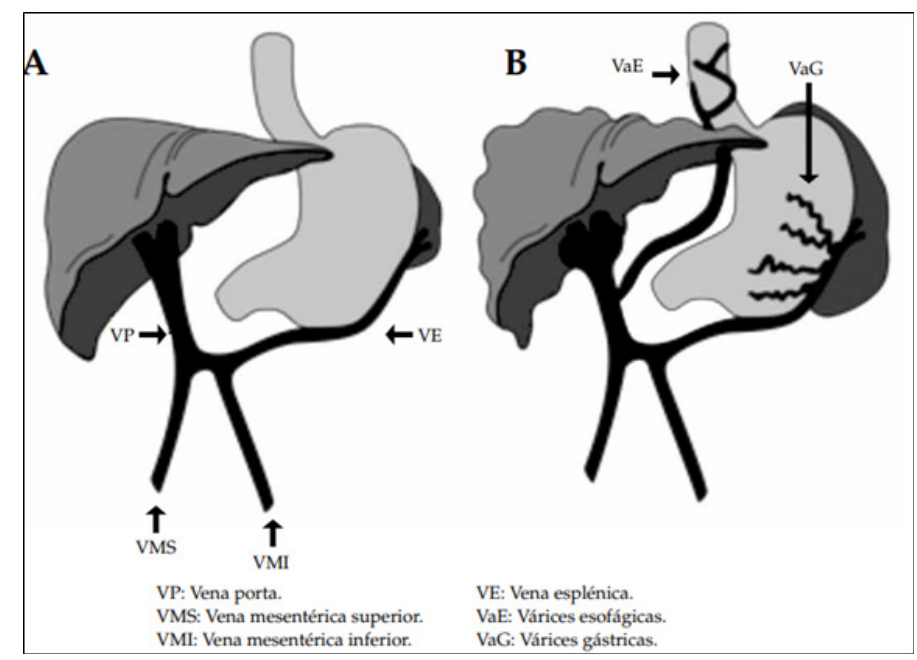

Figura 2. Esquema de la circulación portal. A: sistema porta normal. B: hígado cirrótico: formación de várices y esplenomegalia

Fuente: (Costaguta \& Álvarez) 
La etiología de la Hipertensión Portal, está dada por lo tanto por la presencia de algún obstáculo en la vena porta o sus ramas, o por el incremento del flujo venoso portal causado por una fístula arteriovenosa. Los obstáculos son debidos a una diversidad de causas y asientan a cualquier nivel del sistema portal, por esto los pacientes hipertensos portales sufren dos clases de síntomas: los debidos al aumento de la presión portal que son comunes a todos y los propios de la enfermedad causal que determinan las diferencias clínicas entre los hipertensos portales (Zambrano, 2021).

\begin{tabular}{|c|c|c|}
\hline Prehepaticas & Intrahepáticas & Alteraciones postsinusoidales \\
\hline Trombosis de la vena porta & Alteraciones pre-sinusoidales & Sindrome de Budd-Chiari \\
\hline Sepsis intraabdominal & Cirrosis & Enfermedad venosa ockusiva \\
\hline Pancreatitis crónica & Esquistosomiasis & Pericardits oclusiva \\
\hline Estados protrombóticos & Hiperplasia nodular regenerafva & Falla cardiaca derecha \\
\hline Trombosis de la vena esplénica & Enfermedades mieloproliferativas & Regurgitación tricuspidea \\
\hline \multirow[t]{2}{*}{ Fistula esplánica-arteriovenosa } & Metástasis hepática & \\
\hline & $\begin{array}{l}\text { Enfermedades granulomatosas (tuberculosis, } \\
\text { sarcoidosis) }\end{array}$ & \\
\hline
\end{tabular}

Figura 3. Cuadro Esquemático de la Etiología de la Hipertensión Portal

Fuente: (Pareja, 2016)

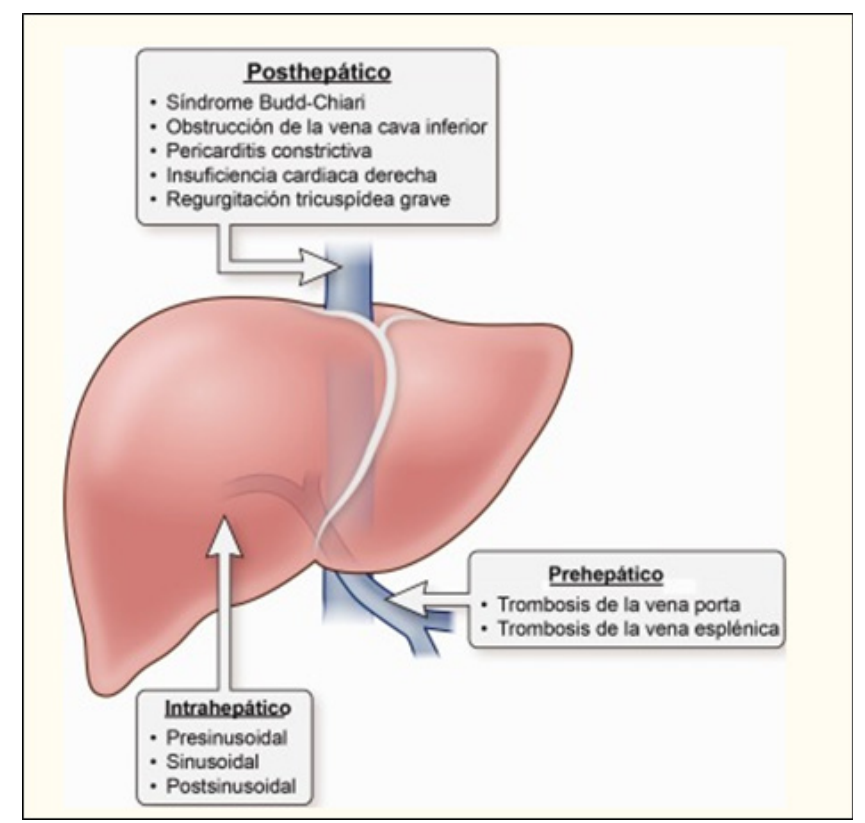

Figura 4. Diagrama que muestra una lista abreviada de las causas prehepáticas, intrahepáticas y posthepáticas de la hipertensión portal.

Fuente: (Koh \& Heller, 2013)

\section{Causas de la hipertensión portal}

La cirrosis es el estadio final de la mayoría de las enfermedades hepáticas crónicas, y la hipertensión portal suele ser el resultado principal y más temprano de la cirrosis. La hipertensión portal cirrótica aparece por 3 mecanismos. El primero es un componente fijo que da lugar a obstrucción mecánica debido a la fibrosis y a la compresión producida por los nódulos regene-

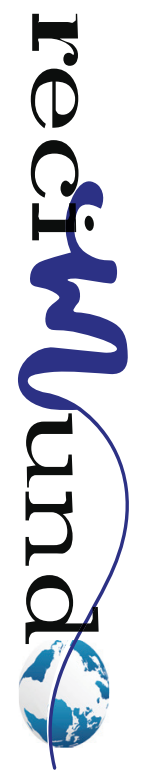


rativos. El segundo, que supone un aumento del $20 \%$ al $30 \%$ de la resistencia intrahepática, es un componente funcional derivado de la contracción activa de las células del músculo liso vascular y las células estrelladas activadas1. El tercer mecanismo consiste en un aumento del flujo sanguíneo portal ocasionado por un aumento de la vasodilatación arterial mesentérica (Koh \& Heller, 2013).

Hipertensión portal no cirrótica: La hipertensión portal también puede desarrollarse en ausencia de cirrosis, en cuyo caso recibe el nombre de HPNC. A diferencia de la hipertensión portal cirrótica, la HPNC se caracteriza por una función de síntesis hepática bien conservada, con aparición menos frecuente de ascitis o encefalopatía hepática (Koh \& Heller, 2013).

La importancia de este síndrome, y su descripción se debe a la gravedad de sus complicaciones: hemorragia digestiva, hiperesplenismo, ascitis, encefalopatía hepática, y síndromes como el hepatopulmonar. La historia natural de la HPT en el niño es difícil de conocer, por diversas patologías que la producen, empleo de tratamientos no controlados, y extrapolación de estudios en adultos a niños, que no se ajusta a los parámetros clínicos y bioquímicas utilizados en los diferentes scores o pautas recomendadas (Nucette, y otros, 2010).

La hipertensión portal (HTP) en los niños es una entidad particularmente desafiante. 1 Debido a la naturaleza previamente intratable de las enfermedades hepáticas terminales en la infancia, la mayoría de las series iniciales sobre HTP pediátrica reportaron la obstrucción prehepática más que las enfermedades hepáticas intrínsecas como causa más frecuente a esta edad. Sin embargo, el conocimiento del espectro clínico de las afecciones que producen HTP y sus consecuencias ha hecho evolucionar muchos conceptos sobre esta entidad. Actualmente se considera que las causas hepáticas representan más del 50 $\%$ dada fundamentalmente por atresia de vías biliares o trastornos metabólicos que involucran al hígado (Hidalgo Marrero, Trinchet So- ler, Manzano Suárez, \& Trinchet Varela, 2010).

En los niños se observa además anemia, esplenomegalia, hepatomegalia, coagulopatías e hiperesplenismo. La complicación más grave es el sangrado por várices esofágicas. Las exploraciones útiles para el diagnóstico son el ultrasonido abdominal y la endoscopia digestiva, entre otras. La biopsia hepática es útil para establecer la causa en pacientes con daño hepático. El tratamiento de la hipertensión portal en la infancia ha cambiado sustancialmente desde los últimos 10 a 20 años. Deben tenerse en cuenta: a) el tratamiento de la enfermedad de base y de la hipertensión portal en sí, b) el tratamiento profiláctico del primer sangramiento variceal, c) el tratamiento del episodio de sangrado variceal, d) la profilaxis del resangrado y d) el tratamiento de otras complicaciones (Andrade Ruiseco, García Pérez, \& Silverio García, 2010).

\section{Métodos diagnósticos en hipertensión por- tal}

\section{Métodos invasivos}

Gradiente de presión venosa hepática (HVPG): Inicialmente se localiza la vena yugular, femoral o cubital a las cuales se les introducirá un catéter guiado por control fluoroscopio que alcanzará la vena hepática principal. Localizado allí y a una distancia de entre 2 a $4 \mathrm{~cm}$ de la vena cava inferior, el catéter medirá la presión venosa hepática libre. Después, en la misma ubicación se insuflará el balón que tiene el catéter generando una obstrucción del flujo sanguíneo. Dicha obstrucción se confirma inyectando medio de contraste que revela un patrón en cuña. Finalmente, tras alcanzar la estabilidad de la presión dentro de la vena hepática, esta se mide determinando la presión venosa hepática en cuña $(1,17)$ (Pareja, 2016).

El gradiente de presión auricular-hepática (HAPG): utiliza la presión auricular derecha en lugar de la presión venosa hepática libre; es un método diagnóstico que busca medir la presión portal de forma similar al HVPG; no obstante, de forma más eficaz (Pareja, 2016). 


\section{Métodos no invasivos}

Elastografía transitoria: La técnica para la medición de la rigidez hepática a través de la elastografía transitoria implica una serie de condiciones del paciente y de los instrumentos a utilizar. Dicha medición se realiza en el lóbulo hepático derecho con el paciente en decúbito dorsal y con el brazo derecho en máxima abducción, para luego localizar un área hepática de al menos $6 \mathrm{~cm}$ de espesor que se encuentre libre de grandes vasos; donde finalmente se realizarán las pruebas para adquirir los datos del examen. Ahora bien, en orden de conseguir información precisa y confiable, se deben obtener 10 disparos válidos del ultrasonido; de manera que se considera falla de la TE si no se alcanza ningún disparo válido y se define de poco confianza si se presentan menos de 10 disparos válidos (Pareja, 2016).

Elastografía por resonancia magnética: La elastografía consiste en estimular mecánicamente el tejido a estudiar mediante compresión estática a través de radiación acústica enfocada o vibraciones de baja frecuencia. Las vibraciones de baja frecuencia se exploran a través de la elastografía por resonancia magnética (MRE - Magnetic resonance elastography), la cual evalúa los campos de ondas en dos o tres dimensiones. De hecho, la MRE es la técnica de elastografía más precisa actualmente utilizada para estadificar el grado de fibrosis hepática. La evidencia actual muestra a la elastografía por resonancia magnética como un medio adecuado para la evaluación de la hipertensión portal y su relación con el desarrollo de várices esofágicas (Pareja, 2016).

ARFI (acoustic radiation force impulse imaging): Es una tecnología de reciente aparición, que provee información sobre la elasticidad de un tejido en tiempo real y que se encuentra incorporado en una unidad convencional de ultrasonido. Se generan pulsos acústicos de aproximadamente $262 \mu$ s, con una frecuencia de 2,67 MHz, lo cual genera ondas de corte en el tejido objetivo del estu- dio. Luego, las velocidades de las ondas de corte en el tejido son medidas en una pequeña área del parénquima en el cual se genera un pequeño desplazamiento (Pareja, 2016).

Fibrotest: El fibrotest es un panel de cinco marcadores séricos relacionados por un algoritmo (a2-macroglobulina, apolipoproteina A-1, haptoglobina, $\gamma$-glutamiltransferasa, bilirrubina sérica) que se encuentra ampliamente validado en términos de su relación con la hipertensión portal grave y que genera un resultado entre 0 y 135 (Pareja, 2016).

\section{Metodología}

La metodología de la investigación, es una revisión bibliográfica, ya que no se van a realizar estudios transversales o de campo más exhaustivo, por ello se ha recurrido a la investigación por medios electrónicos como fuente primaria de obtención de información, revistas, páginas web, libros de investigación, entre otros. Que han dado aportes claves para la consecución de este trabajo.

\section{Resultados}

\section{Fisiopatología}

La cirrosis es la causa más común de hipertensión portal y la principal indicación de trasplante hepático en el mundo. En ella, las alteraciones estructurales y funcionales que conducen a una franca disfunción endotelial que aumentan el tono y la resistencia vascular intrahepática explican la aparición de la hipertensión portal. Dichas alteraciones determinan el aumento en un $25 \%$ de la resistencia vascular. Los cambios en la circulación intra y extrahepática son fundamentales en la fisiopatología de la hipertensión portal. Con respecto al primero, los eventos resultantes de la disfunción endotelial, como las alteraciones de los mecanismos regulatorios vasomotores y la inflamación propia del daño hepático, conducirán al desarrollo de fibrosis y desarrollo de nódulos de regeneración que comprometerán la arquitectura intrahepática (Pareja, 2016). 
En circunstancias normales no se visualizan colaterales y se observa una buena arborización en el hígado. Cuando son evidentes las colaterales, se confirma el diagnóstico de hipertensión portal. Por lo general con esta técnica se puede definir la vena coronaria estomáquica que contribuye a las várices esofágicas. También se ha empleado la vena umbilical para delimitar el sistema portal. En $80 \%$ de estos casos se puede aislar la vena obliterada y dilatarse para introducir un catéter e inyectar una sustancia radiopaca en la vena porta izquierda. También se pueden visualizar las venas porta y hepática mediante canulacion transhepática percutánea (Zambrano, 2021).

Los sistemas colaterales más importantes son el sistema coronario-ácigos causantes de las várices esofágicas y esofagogástricas, el sistema umbilical de la pared abdominal que puede producir el síndrome de Ruveilhier-Baumgarten (venas colaterales muy grandes y tortuosas, que se asocian con murmullo), las venas Retzius, que representan conexiones portocavales en la región posterior o retroperitoneal; las venas Sappey, que son conexiones entre la cara posterior superior del hígado, el diafragma y el pericardio; y por último el sistema hemorroidal, superior, medio e inferior, causantes de las hemorroides internas y externas (Zambrano, 2021).

Los sistemas colaterales más importantes son el sistema coronario-ácigos causantes de las várices esofágicas y esofagogástricas, el sistema umbilical de la pared abdominal que puede producir el síndrome de Ruveilhier-Baumgarten (venas colaterales muy grandes y tortuosas, que se asocian con murmullo), las venas Retzius, que representan conexiones portocavales en la región posterior o retroperitoneal; las venas Sappey, que son conexiones entre la cara posterior superior del hígado, el diafragma y el pericardio; y por último el sistema hemorroidal, superior, medio e inferior, causantes de las hemorroides internas y externas (Zambrano, 2021).

Aunque el volumen de sangre que llevan estas colaterales es a veces, muy elevado nun- ca puede quedar del todo aliviada la hipertensión portal, debido a que el estímulo para el desarrollo de estas colaterales es la misma hipertensión portal. En el aspecto clínico existe una cierta correlación directa entre el tamaño de las várices esofágicas y el nivel de la hipertensión portal (Zambrano, 2021).

Algunos autores han postulado que la causa primaria de la hipertensión portal, al menos en algunas enfermedades, es una elevación del flujo arterial esplácnico, ya sea directamente, ya sea a través de cortocircuitos arteriovenosos en el bazo o en la submucosa del estómago y del intestino delgado (Zambrano, 2021).

A consecuencia de la hipertensión, asociada con la misma, es entonces que congestionan las vías colaterales ya mencionadas, produciéndose como vimos várices esofagogástricas que pueden ser causa de hemorragia. Todavía es motivo de discusión si las hemorragias dependen de aumentos bruscos de la presión portal o se deben a traumatismos o esofagitis péptica, o lo más probable a la combinación de más de una de estas causas (Zambrano, 2021).

La hipertensión portal, a consecuencia fundamentalmente de la hiperpresión vascular a nivel de la sinusoide hepática, condiciona la aparición de ascitis. El aumento de la presión del eje venoso portal, incrementándose de este modo la presión en la vena esplénica, condiciona un estasis venoso y secuencialmente una congestión pasiva esplénica, que puede llevar a un hiperesplenismo, entendiéndose como tal aumento de todas las funciones del bazo, que a su vez produce anemia, leucopenia y plaquetopenia. El deterioro progresivo de la función hepática puede llevar a la hiperamonemia, encefalopatía y coma hepático (Zambrano, 2021).

\section{Conclusiones}

Las personas que padecen de cirrosis, tienen una alta probabilidad de padecer también la hipertensión portal, esto agrava aún más el cuadro del paciente, posibilitando su muerte. 
Hay diferentes elementos moleculares, vasomotores y de flujo sanguíneo que son los causantes de la hipertensión portal.

El estudio de la fisiopatología de la hipertensión portal, ha evolucionado los últimos años. La obstrucción del flujo venoso portal, por los cambios en la arquitectura del hígado y vasodilatación esplanica y sistémica. Hacia un estado hemodinámico, regulado por componentes neurales, celulares y humorales, que actúan de manera endocrina, paracrina y autocrina.

En la actualidad hay diferentes tipos de mecanismos de diagnóstico de la hipertensión portal. Uno de los más utilizados y confiables es el gradiente de presión venosa hepática (GPHV), que aunque es un método invasivo, sigue siendo el más sensible y específico, para su diagnóstico. Dentro de los métodos no invasivos se encuentran la elastografía transitoria, que es muy eficaz y seguro para la detección de la hipertensión portal. El problema es que necesita de valores significativos de la presión portal para poder evaluar el grado de hipertensión como un método de seguimiento y falta de más estudios comparativos. Por otro lado, la elastografía por resonancia magnética, el fibrotest y ARFI, son métodos no invasivos, pero requieren un valor ( $>12$ $\mathrm{mm} \mathrm{Hg}$ ), para su diagnóstico, lo que la limitan en su uso cotidiano en la evaluación de pacientes con hepatopatías crónicas.

En la población infantil la hipertensión portal más frecuente es la prehepatica. Las causas pueden ir, desde cateterismo umbilical, así como infecciones neonatales umbilicales, sepsis generalizada o episodios diarreicos con deshidratación. Sin embargo estudios de otros autores han revelado que la hipertensión portal hepática, se puede presentar entre el 25 y $45 \%$ de los niños.

\section{Bibliografía}

Álvarez, B. G. (2002). Hipertensión portal. In Anales de Cirugía Cardíaca y Vascular, 8(1), 46-55.

Andrade Ruiseco, M., García Pérez, W., \& Silverio García, C. (2010). Hipertensión portal en niños: análisis de 20 años de trabajo. Revista Cubana de Pediatría, 82(1), 0-0.

Costaguta, A., \& Álvarez, F. (s.f.). Hipertensión portal en pediatría. I: Aspectos fisiopatológicosy clínicos. Archivos argentinos de pediatría, 108(3), 239-249.

Hidalgo Marrero, Y., Trinchet Soler, R., Manzano Suárez, J., \& Trinchet Varela, C. (2010). Hipertensión portal prehepática en los niños. Revista Cubana de Pediatría, 82(4), 76-88.

Ibarrola-Calleja, J. L., Núñez, F., Rodríguez, M., \& Ordóñez-Céspedes, J. (2011). Hipertensión portal. Acta Médica Grupo Ángeles, 9(2), 83-91.

Koh, C., \& Heller, T. (16 de 10 de 2013). doi:10.1002/ cld.269

Montaño-Loza, A., \& Meza-Junco, J. (2005). Patogénesis de la hipertensión portal. Revista de investigación clínica, 57(4), 596-607.

Nucette, A., Navarro, D., Colina, N., López, K., Durango, R., Arrieta, A., \& García, G. (2010). Hipertensión portal en niños: historia natural, evolución, tratamiento y pronóstico. Gen, 64(4), 323-329.

Pareja, J. S. (2016). Métodos diagnósticos en hipertensión portal. Revista Colombiana de Gastroenterología, 31(2), 135-145.

Zambrano, T. (25 de 01 de 2021). http://medicosecuador.com/. Obtenido de http://medicosecuador. com/librosecng/articuloss/3/hipertension_portala. $\mathrm{htm}$

\section{CITAR ESTE ARTICULO:}

Muñoz Andrade, L. A., Mera Sabando, A. J., \& López Álvarez, E. Y. (2021). Aspectos fisiopatológicos en la hipertensión portal. RECIMUNDO, 5(1), 42-49. https://doi. org/10.26820/recimundo/5.(1).enero.2021.42-49 